\title{
Identification of stiffness and damping characteristics of axial air-foil bearings
}

\author{
Vikas Arora • P. J. M. van der Hoogt • \\ R. G. K. M. Aarts $\cdot$ A. de Boer
}

Received: 21 January 2011 / Accepted: 22 June 2011 / Published online: 5 July 2011

(C) The Author(s) 2011. This article is published with open access at Springerlink.com

\begin{abstract}
Air-foil bearings (AFBs) are self acting hydrodynamic bearings made from sheet metal foils comprised of at least two layers. The innermost "top foil" layer traps a gas pressure film that supports a load while the layer or layers underneath provide an elastic foundation. AFBs are currently used in many commercial applications, both terrestrial and in aerospace. AFBs provide a means to eliminate the oil system leading to reduce weight and enhanced temperature capability. Regardless of the application of the AFBs, the identification of the structural characteristics is important for successful design practice. In the present work, structural characteristics (stiffness and damping) of axial AFBs are indentified in the light of experimental results. Due to the initial high torque requirement of the $\mathrm{AFB}$, the experimental setup using a single AFB is proposed instead of standard two-foil bearing setups. Experiments are carried out at maximum speed of 60,000 rpm. Sub-structuring approach
\end{abstract}

V. Arora $(\square)$

Department of Mechanical and Structural Engineering and Material Science, Universitetet i Stavanger,

Stavanger, Norway

e-mail: vikas.arora@uis.no

P. J. M. van der Hoogt · A. de Boer

Structural Dynamics and Acoustics, University of Twente, Enschede, The Netherlands

R. G. K. M. Aarts

Mechanical Automation, University of Twente, Enschede, The Netherlands is used for identification of the structural (stiffness and damping) characteristics of the AFB. The results have shown that the developed experimental procedure is able to identify the stiffness and damping characteristics of axial AFBs accurately.

Keywords Axial air-foil bearings . Stiffness and damping identification . Sub-structuring $\cdot$ High speed

\section{Introduction}

Air-foil bearings (AFBs) are currently used in many commercial applications, both terrestrial and in aerospace. AFBs provide a means to eliminate the oil system leading to reduce weight and enhanced temperature capability. AFBs are complaint-surface use ambient air as the lubricant fluid. A hydrodynamic pressure builds up within the small gap or film between rotating shaft and the top foil. Figure 1 shows the configuration of a "first generation" bump type AFB. The AFB consists of a thin (top) foil and a series of corrugated bump strip supports. The leading edge of the top foil is free, and the foil trailing edge is welded to the bearing housing. Beneath the top foil, a bump structure is laid on the inner surface of the bearing. The top foil of the smooth surface is supported by a series of bumps acting as springs, 


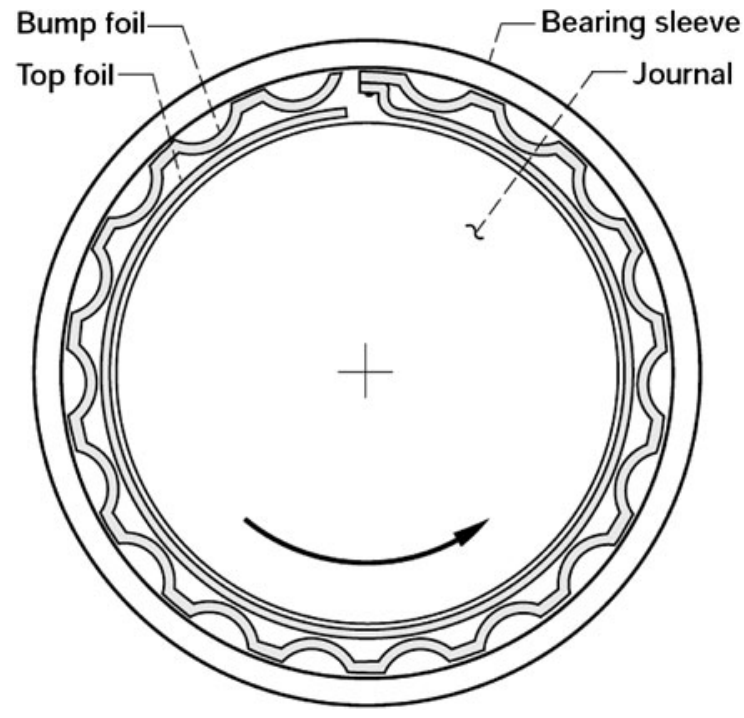

Fig. 1 Compliant air-foil journal bearing

thus making the bearing complaint. The bump foil layer gives the bearing flexibility that allows it to tolerate significant amounts of misalignment and distortion that would otherwise cause a rigid (oil lubricated) bearing to fail. In addition, micro-sliding between top foil and bump foil generates coulomb damping which can increase the dynamic stability of the rotor-bearing system. During normal operation of the foil bearing supported machine, the rotation of the rotor generates a pressurized gas film that pushes the top foil out radially and separate the top foil from the surface of the rotating shaft. The pressure in the air film is proportional to the relative surface velocity between the rotor and top foil. Thus, faster the rotor rotates, the higher the pressure, and the more is the load the bearing can support. When the rotor first begins to rotate, the top foil and the rotor surface are in contact until the speed increases to a point where the pressure in the air film is sufficient to push the top foil away from the rotor and support its weight. This threshold speed is generally referred as liftoff speed. Likewise, when the rotor slows down to a point where the speed is insufficient to support the rotor weight, the top foil and rotor again come in contact. Axial AFB is shown in Fig. 2 is composed of similar elements used in the radial AFBs, but are designed to support a shaft axially. Often discrete complaints pads are attached circumferentially around annular backing plate and consist of a complaint reinforcing structure supporting the flexible top foils. The rotating thrust runner drags the air into converging gap at each pad in a manner functionally similar to rotating radial bearing. In both axial and radial bearings, the hydrodynamic gas film is fully formed only above a threshold speed.

The dynamic behavior of a rotating system is significantly influenced by the structural (stiffness

Fig. 2 Axial AFB

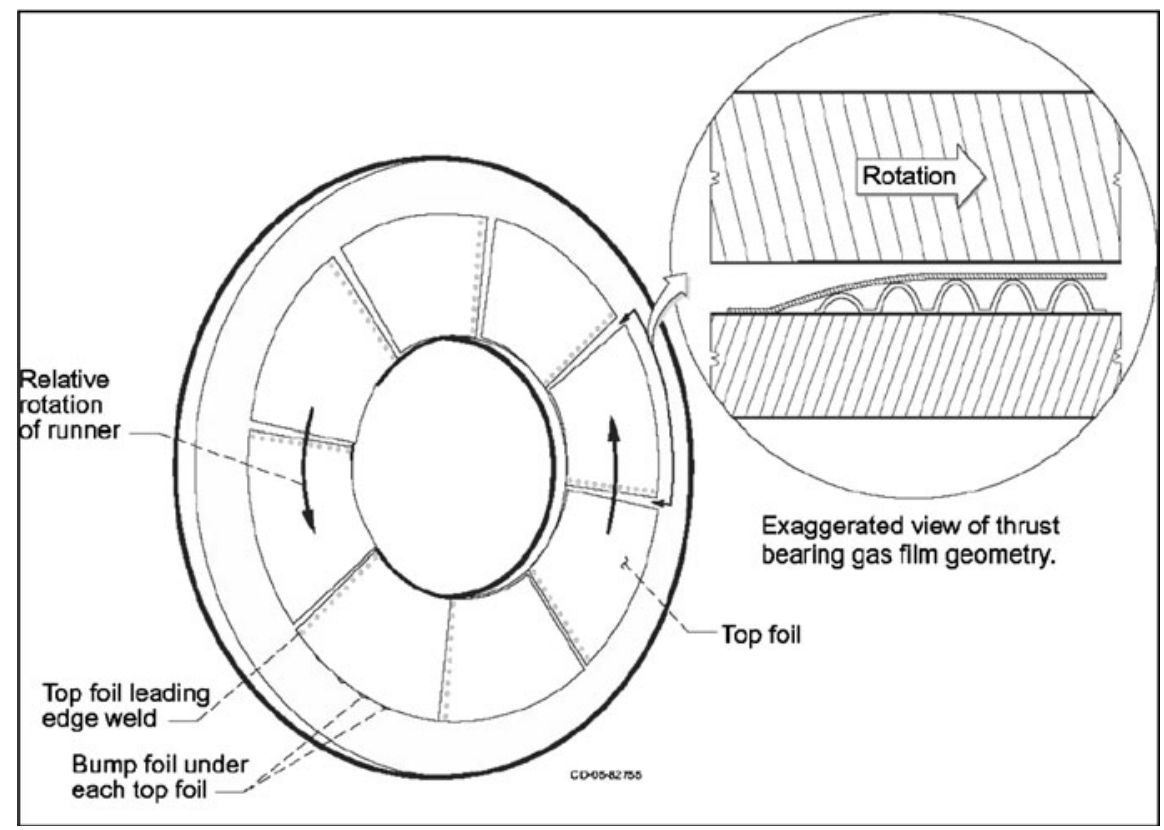


and damping) characteristics of the bearings. The exact values of the stiffness and damping coefficients of AFBs are difficult to predict. The identification of structural characteristics of AFBs can be classified broadly into two categories:

1. Mathematical route

2. Experimental route

The geometric configuration of AFB is very complicated and numerical calculations of various AFB characteristics are difficult and also they do not predict accurately the dynamics of complete supportrotor-bearing system Therefore, the mathematical route uses empirical values, mainly film thickness and dry friction coefficients, to develop mathematical models. The mathematical models of AFBs developed using empirical values do not predict the behavior of complete rotor-bearing system at different rotating speeds. The determination of stiffness and damping in AFB is a difficult task and optimization techniques provide an alternative technique for identification. In the optimization technique, finite element model is corrected in the light of experimental data. Prediction capabilities of the rotorbearing systems can be improved significantly by identifying the dynamic characteristics of the AFB using optimization techniques. Hence, in this work, firstly a detail is given regarding the mathematical techniques, which uses empirical values, and then emphasis will be on the optimization techniques.

$\mathrm{Ku}$ and Heshmat (1993) presented an analytical procedure to identify the dynamic stiffness and the equivalent viscous damping of AFBs. A simple analytical model assembles individual bump stiffnesses and renders predictions for the AFB structural stiffness as a function of the bump geometry and material, dry-friction coefficient, load orientation, clearance and preload. The dry-friction coefficient and clearance values are obtained from the experiment. Ku (1993) described an experimental investigation to characterize the structural dynamic force coefficients of corrugated bumps used in foil bearings. Dynamic force perturbations are imposed to a sixbump strip under different test conditions and various bump geometrical configurations using dry friction coefficients ranging from 0.4 to 0.6 , depending on the surface coating. Heshmat and $\mathrm{Ku}$ (1994) developed an experimental procedure to identify the structural stiffness and equivalent viscous damping coefficient by exciting, with two electromagnetic shakers, a nonrotating shaft supported on AFBs. This procedure uses empirical values of clearance and dry friction for predicting stiffness and damping characteristics of AFB at different rotational speeds. An analytical model (Ku and Heshmat 1994a, b), uses bumps curvature effect, force interaction between bumps, and the dry friction coefficient ranging from 0.4 to 0.6 for predicting stiffness and damping characteristics. Salehi et al. (2003) performed dynamic force tests on corrugated metal sheets (bump foil strips). Dynamic force excitations are exerted on the bump strip using an electromagnetic shaker at various load and frequency conditions. Equivalent viscous damping coefficients and dry friction forces are extracted from the resulting hysteresis loops (force versus displacement) for various test conditions. DellaCorte and Valco (2000) introduced a simple "rule of thumb" method to estimate the load capacity in foil gas journal bearings. The method relates the bearing load capacity to the bearing size and the speed through an empirically based load capacity coefficient. Rubio and San Andrés (2006) estimated the dry friction coefficients from shaker-exerted dynamic load tests. It can be observed from the above references that stiffness and damping characteristics are predicted by using mathematical model of the AFB and empirical values of clearance and dry friction coefficients. These empirical values used in the mathematical models do not predict the rotor-bearing systems completely at different rotating speeds. Prediction capabilities of the rotor-bearing systems can be improved significantly by identifying the dynamic characteristics of the system using optimization techniques. The optimization techniques, correct the finite element model in the light of experimental data. The state of the art of the optimization techniques for identification of structural characteristics are presented in the section below.

An important application of the optimization procedure in mechanical system is devoted to inverse problems. The determination of the structural properties of bearings is a difficult task and optimization techniques provide an alternative way for the identification of structural properties of bearings. Some researchers have focused on using an optimization scheme to correct the finite element model to determine the objective function that quantifies the difference between experimental data and analytical data (Arora et al. 2009a, b). During the past decade, many research 
works have dealt with identification of structural characteristics of bearings and rotor-bearing systems using optimization techniques. These optimization techniques require correction of the finite element model of the rotating system in the light of experimental data. Lee and Hong (1998) proposed an identification scheme for estimation of structural characteristics of bearings from out of balance response. Chen and Lee (1995) proposed a new method of estimating coefficients of bearings. Edwards et al. (2002) identified the unbalance and support parameters of flexible rotor-bearings-foundation system from single rundown. Jiang and Yu (2000) studied the identification of dynamic characteristics of journal and axial bearings. Tiwari et al. (2002) identified speed-dependent bearing parameters using unbalance response measurement for multi-degree-of freedom flexible rotor-bearing system. Kim et al. (2007) identify the bearing characteristics using genetic algorithm. The procedure can identify not only unknown bearing stiffness but also unbalance information of the disk.

The AFB requires a high initial torque. San Andrés and Kim (2008) developed an experimental setup, which uses two motors, one starting motor and one high speed motor. The starting motor assists in start up the rotor and operating is shifted to the high speed motor after starting through a centrifugal clutch. Kim and Park (2009), Isomura et al. (2005) and Belforte et al. (2006) developed experimental setup using a high torque turbine for testing the airfoil bearings.

In this paper, an experimental setup is proposed which uses a single one AFB. The use of only one AFB will reduce the initial torque requirement as well as cost of an experimental setup significantly and also it will not affect the credibility of the experimental results. As in this paper, the objective is to find the structural characteristics of the AFB. The use of one AFB can not predict the behaviour of complete rotor system. The natural frequencies of the complete rotor-bearing system are affected by the structural characteristics of the AFB only. So in this proposed experimental setup, the natural frequencies are only affected by a single AFB and sub-structuring technique has been used for identification of structural characteristics of AFB at operating different speeds. The identification algorithm, which uses the vibration data (natural frequencies and mode shapes) of the complete rotor system, for identifying structural characteristics of the AFBs has also been developed. This identification technique also requires developing a sub-structuring procedure.

\section{Theory}

In this section, a structural characteristics identification procedure and algorithm is presented. The structural identification procedure is based on the sub-structuring technique. In the sub-structuring technique, each component is considered in isolation. The structural properties of the isolated structure are identified using experimental data. All the isolated components are subsequently assembled to make complete rotor-bearing-support system. The identification algorithm is then applied to complete system to identify the structural characteristics of AFBs. The identification algorithm used in the present work, is an inverse eigensensitivity method. In the inverse eigensensitivity method, the unknown bearing stiffness value is determined by using eigenvalue sensitivity. So the identification consists of two steps: substructuring and applying an identification algorithm for identification of stiffness of AFB.

\subsection{Sub-structuring}

The schematic diagram of the rotor-bearing-support system is shown in the Fig. 3. It can be observed from the Fig. 3 that the rotor-bearing-support system consists of five components, namely the motor support, motor, rotor, axial AFB and axial bearing support. The structural characteristics of the isolated individual components, except the axial AFB, is identified/ corrected in light of experimental data as shown in Fig. 4. All the components are subsequently assembled to form complete rotor-bearing-support system. The AFB characteristics are identified in the last step. The rotor-bearing-support system can be written in terms of dynamic stiffness matrices of its constituent components as:

$$
\begin{aligned}
& Z_{R} u_{R}=f_{R} \\
& Z_{B} u_{B}=f_{B} \\
& Z_{S} u_{S}=f_{S}
\end{aligned}
$$

where $\mathrm{Z}$ is the subscript-dependent dynamic stiffness matrix of the local system component, $u$ is the subscriptdependent response vector and $\mathrm{f}$ is subscript dependent 


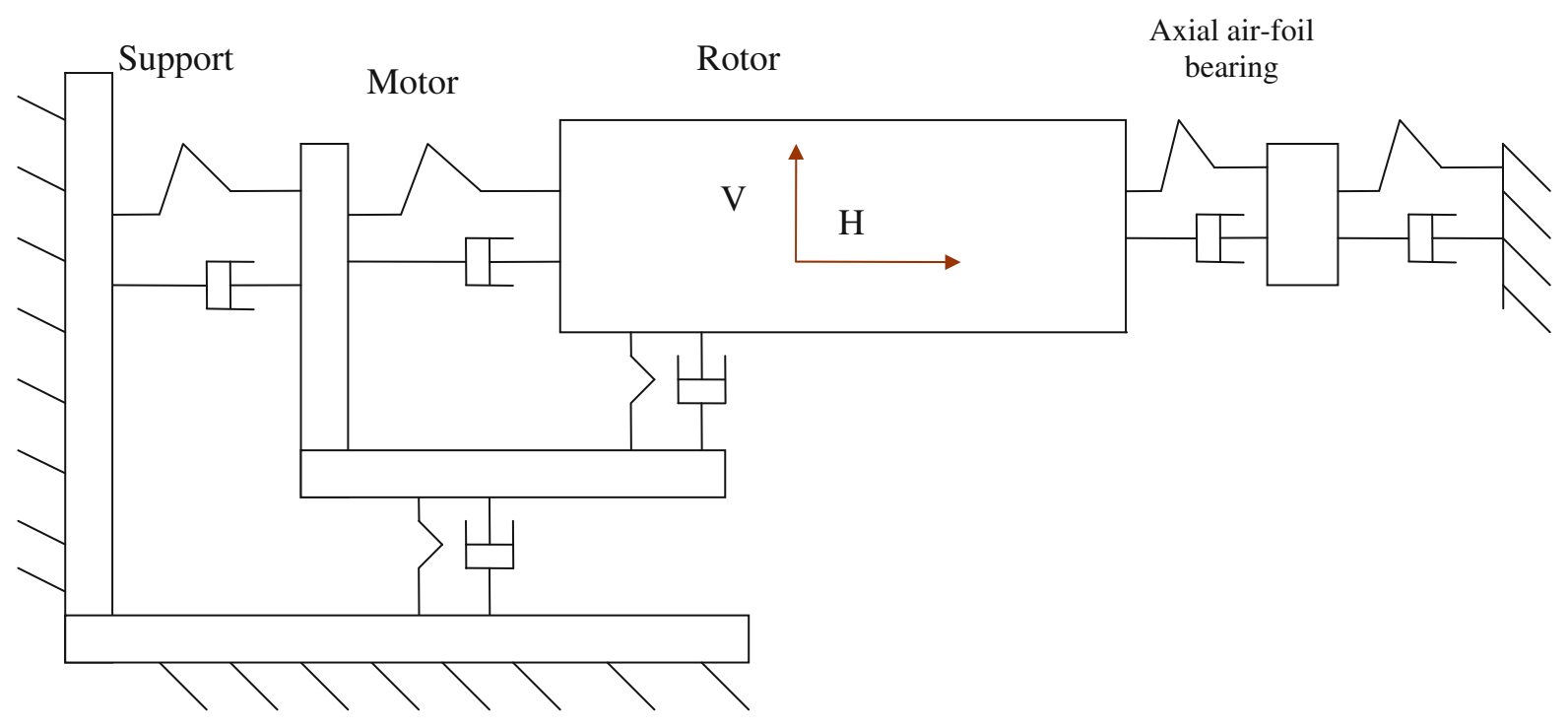

Fig. 3 Schematic diagram of rotor-bearing system

force. Subscripts R, B, S relate to rotor, bearings and supports (motor support, motor and bearing support, in this case) respectively. The individual dynamic stiffness matrices of each component are:

$Z_{R}(\omega)=K_{R}-\omega^{2} M_{R}+j \omega C_{R}$

$Z_{B}(\omega)=K_{B}+j \omega C_{B}$

$Z_{S}(\omega)=K_{S}-\omega^{2} M_{S}+j \omega C_{S}$

where K, M, C are the subscript-dependent stiffness, mass and damping matrices. The equations presented in the sub-components of rotor-dynamic system are combined to form the whole system as:

$$
\left[\begin{array}{cc}
Z_{R}+Z_{B} & -Z_{B} \\
-Z_{B} & Z_{B}+Z_{S}
\end{array}\right]\left\{\begin{array}{l}
u_{R} \\
u_{S}
\end{array}\right\}=\left\{\begin{array}{l}
f \\
0
\end{array}\right\}
$$

It can be noticed from Eq. 7 that the rotor-bearingsupport system is portioned into rotor, bearing and support components to identify the dynamic characteristics of the rotor-bearing-support system. The goal of partition is to use the eigendata of each component separately to identify the dynamic characteristics of each component.

\subsection{Identification algorithm}

The identification algorithm is based on the inverse eigensensitivity method (Modak et al. 2000). This method uses eigendata namely eigenvalues, eigenvectors and damping ratios, which are obtained by modal analysis of measured FRFs. The updating parameters corresponding to the finite element model are corrected to bring FE eigendata closer to that of experimentally derived ones. Most often updating equations is based on a linear approximation of the modal data that is generally a nonlinear function of the updating parameters. The linearization of $\mathrm{rth}$ eigenvalue $\lambda^{r}$ gives:

$\lambda_{X}^{r}=\lambda_{A}^{r}+\sum_{i=1}^{n u}\left(\frac{\partial \lambda_{A}^{r}}{\partial p_{i}} \Delta p_{i}\right)$

where subscripts $\mathrm{A}$ and $\mathrm{X}$ denote analytical and experimental respectively. $\mathrm{p}$ is the updating parameter and nu is number of updating parameters. Eigen sensitivities are represented by derivatives, can be calculated by from relationship given by Fox and Kapoor (1968) as:

$\frac{\partial \lambda_{A}^{r}}{\partial p_{i}}=\{\varphi\}_{A}^{r T}\left[\frac{\partial K}{\partial p_{i}}-\lambda_{A}^{r} \frac{\partial M}{\partial p_{i}}\right]\{\varphi\}_{A}^{r}$

where $\{\varphi\}_{A}^{r}$ is the rth mass-normalized eigenvector. Using Eq. 9, Eq. 8 can be written for the chosen $m$ number of modes. These equations together, after dividing and multiplying by $\mathrm{p}_{\mathrm{i}}$ and then writing $\mathrm{u}_{\mathrm{i}}$ in place of $\Delta p_{i} / p_{i}$ can be written in the following matrix form: 
Fig. 4 Flow chart of proposed identification procedure for AFB

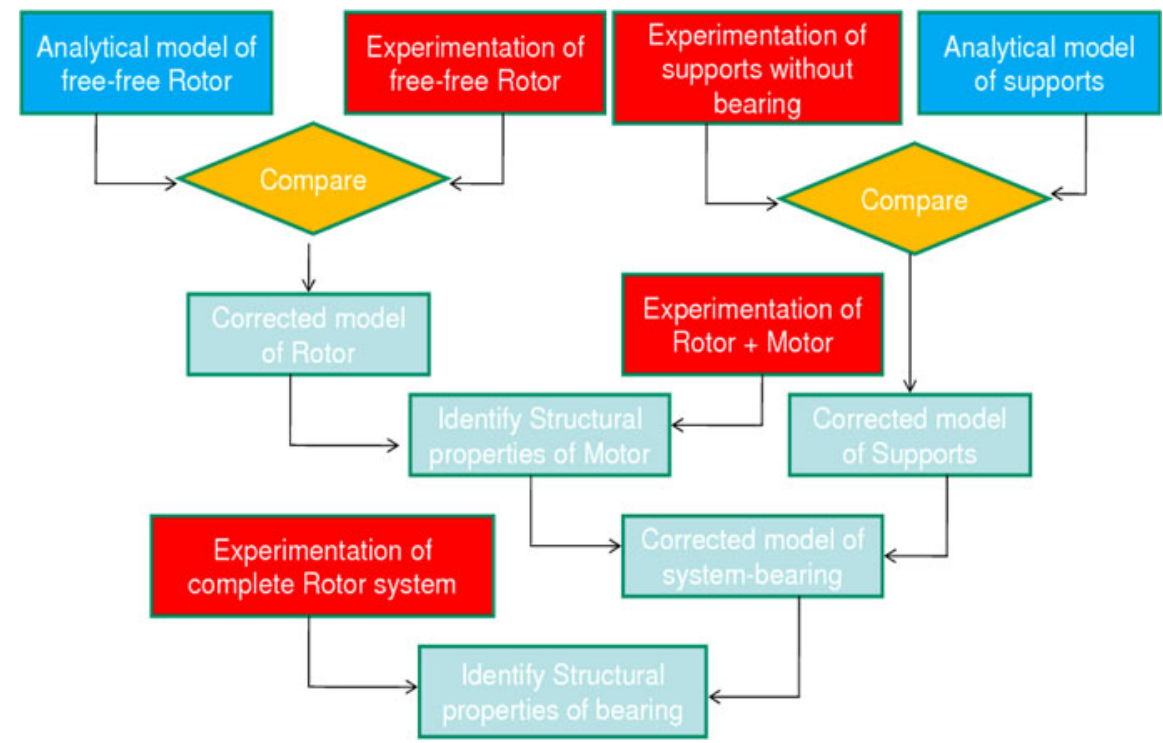

$$
\begin{aligned}
& {\left[\begin{array}{cccc}
p_{1} \cdot \frac{\partial \lambda_{A}^{1}}{\partial p_{1}} / \lambda_{A}^{1} & p_{2} \cdot \frac{\partial \lambda_{A}^{1}}{\partial p_{2}} / \lambda_{A}^{1} & \ldots & p_{n u} \cdot \frac{\partial \lambda_{A}^{1}}{\partial p_{n u}} / \lambda_{A}^{1} \\
p_{1} \cdot \frac{\partial \lambda_{A}^{2}}{\partial p_{1}} / \lambda_{A}^{2} & p_{2} \cdot \frac{\partial \lambda_{A}^{2}}{\partial p_{2}} / \lambda_{A}^{2} & \ldots & p_{n u} \cdot \frac{\partial \lambda_{A}^{2}}{\partial p_{n u}} / \lambda_{A}^{2} \\
\ldots & \ldots & \ldots & \cdots \\
p_{1} \cdot \frac{\partial \lambda_{A}^{m}}{\partial p_{1}} / \lambda_{A}^{m} & p_{2} \cdot \frac{\partial \lambda_{A}^{m}}{\partial p_{2}} / \lambda_{A}^{m} & \ldots & p_{n u} \cdot \frac{\partial \lambda_{A}^{m}}{\partial p_{n u}} / \lambda_{A}^{m}
\end{array}\right]} \\
& \quad \times\left\{\begin{array}{c}
u_{1} \\
u_{2} \\
\cdot \\
u_{n u}
\end{array}\right\}=\left\{\begin{array}{c}
\left(\lambda_{X}^{1}-\lambda_{A}^{1}\right) / \lambda_{A}^{1} \\
\left(\lambda_{X}^{2}-\lambda_{A}^{2}\right) / \lambda_{A}^{2} \\
\\
\left(\lambda_{X}^{m}-\lambda_{A}^{m}\right) / \lambda_{A}^{m}
\end{array}\right\}
\end{aligned}
$$

The above equation can be represented in a compact form as:

$$
[S]_{m \times n u}\{u\}_{n u \times 1}=\{\Delta e\}_{n u \times 1}
$$

where $\mathrm{S}$ is the sensitivity of eigenvalues with respect to the updating parameters and $\{\Delta e\}$ is a vector of difference between experimental and analytical eigenvalues. The above matrix equation is solved for $\{u\}$ using the routine for finding the pseudo-inverse of a matrix available in MATLAB. The pseudo-inverse, calculated by the routine using singular-value decomposition of a matrix, is related to the least-squares problem, as the value of $\{u\}$ that minimizes $\|[S]\{u\}-$ $\{\Delta e\} \|^{2}$ can be given by $\{u\}=[S]^{+}\{\Delta e\}$. The superscript ' + ' denotes pseudo-inverse. The $\{u\}$ so found is used to update the vector of physical variables $\{p\}$, stiffness, and then the updated version of the analytical finite element model is built using the new values of stiffnesses. The process is repeated in an iterative way until convergence is achieved. To estimate the damping ratio from the frequency domain, the half-power bandwidth method is used in the present study.

\section{Experimental setup}

The initial torque requirement for AFBs is very high. To overcome this problem, an experimental setup is designed which uses a single AFB instead of standard two AFBs. In this section, the design of the experimental setup is given in detail. It includes the selection of the motor, analysis of the rotor, design of bearing supports and the design of the motor support. In this section, details of each component are given.

\subsection{Selection of the motor}

The torque required to rotate the AFB supported rotor is greatest when the rotor is in contact with the stator before there is sufficient speed to generate a fluid film separating the two surfaces. To initiate rotation sufficient torque must be applied to the rotor to overcome the sliding frictional forces in the AFBs, the rotor inertia and the required rotational acceleration. Once sliding begins the torque requirement to sustain rotation drops since the kinematic coefficient of friction is typically less than the static coefficient of friction. Upon development of the fluid film and rotor 
Fig. 5 a Torque and b power characteristics of the selected motor

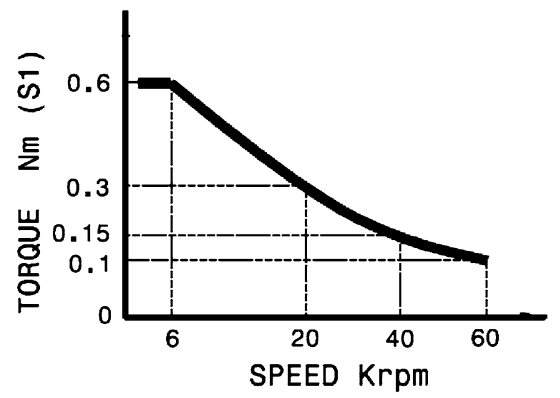

(a)

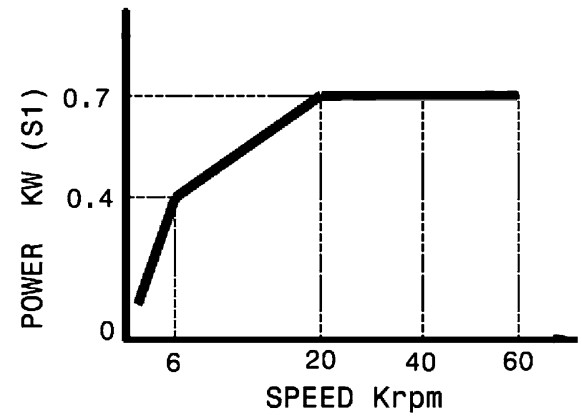

(b) lift-off the torque requirement for rotation will further decline. Due to the initial high torque requirement, researchers have used a high speed and torque turbines (Kim and Park 2009; Isomura et al. 2005; Belforte et al. 2006) or a separate high torque starting motor (San Andrés and Kim 2008) and high speed rotor. Both types of experimental setups are expensive. In order to reduce the cost of experimental setup, the experimental setup is designed such that a single AFB can be used instead of two bearings. The torque and power characteristics of the selected motor are given in Fig. 5. It can be noticed from Fig. 5a that the initial torque of the selected motor is $0.6 \mathrm{Nm}$ and it can run up to a speed of $60,000 \mathrm{rpm}$. It is very important to know that the first natural frequency of the shaft is far above the operational speed of the motor shaft. The modal analysis of the motor was also carried out to find out the natural frequency of the motor shaft. The motor shaft was excited by an impact hammer and the response was measured using an accelerometer. A response plot of the motor is shown in the Fig. 6. It can be observed from Fig. 6 that the first bending natural frequency of the motor shaft is above $3,000 \mathrm{~Hz}$ which is far above the operation speed of 1,000 rps. The axial and radial stiffness of the whole motor system was identified in light of the experimental results, which comes out to be $2 \times 10^{10}$ and $1.6 \times 10^{11} \mathrm{~N} / \mathrm{m}$, respectively.

\subsection{Rotor}

The rotor was designed to have its first natural frequencies above the operating speed of the system. The details of the rotor are shown in Fig. 7. It can be observed from Fig. 7 that the rotor is a stepped rotor and is hollow. The finite element of the rotor was built both on MATLAB using beam elements. The lowest natural frequencies of the free-free rotor are

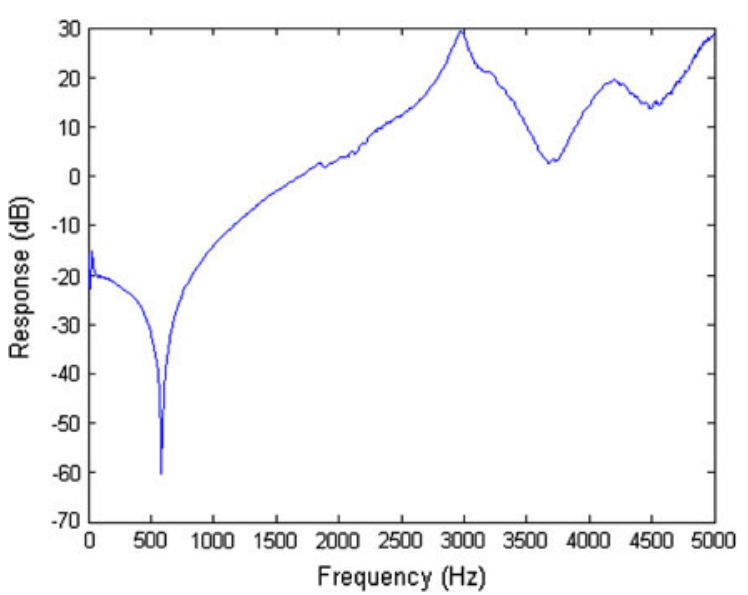

Fig. 6 Response of motor shaft

given in Table 1. It can be noticed from the Table 1 that the first natural frequency is far above the proposed operating speed of the rotor. The experimental modal analysis of the free-free rotor was carried out to validate the FE model results of the rotor. The free-free condition is achieved by hanging the rotor in flexible strings. The modal test is performed by exciting the rotor with an impact hammer and the response is measured at one location using an accelerometer. The measurements on the rotor were carried out at three different locations spaced equally along the length of the shaft as shown in Fig. 7. The first natural frequency of the rotor is $4,200 \mathrm{~Hz}$, which is very close to the natural frequency predicted by FE model.

\subsection{Motor support}

The motor support has been designed and manufactured. The solid model of the motor support is given in Fig. 8. It can be observed from Fig. 8 that the 


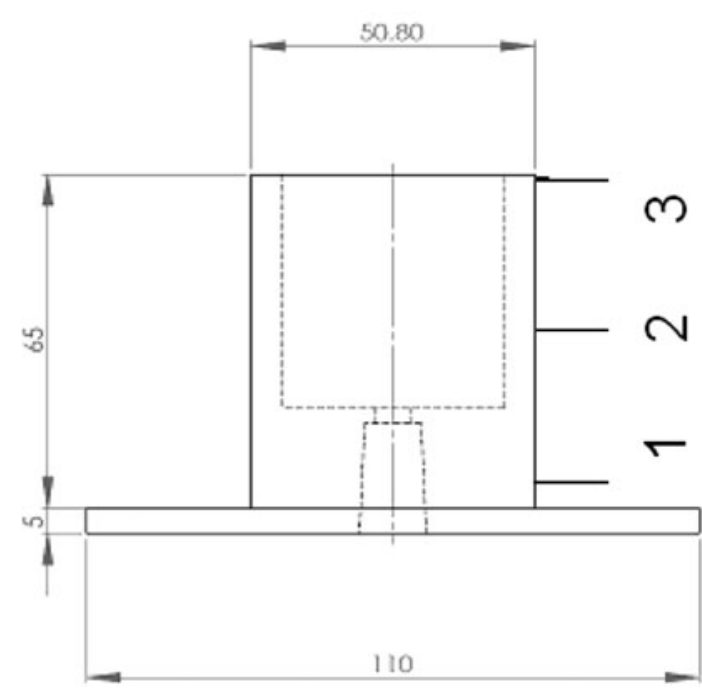

Fig. 7 Details of the rotor and measurement points on the rotor

Table 1 Natural frequencies of the rotor using FE model

\begin{tabular}{ll}
\hline Mode number & Natural frequency $(\mathrm{Hz})$ \\
\hline 1. & 0 \\
2. & 0 \\
3. & 0 \\
4. & $5.0 \mathrm{e}-003$ \\
5. & $6.8 \mathrm{e}-003$ \\
6. & $1.1 \mathrm{e}-002$ \\
7. & 4360.9 \\
8. & 4386.8 \\
9. & 4528.1 \\
10. & 4714.7 \\
\hline
\end{tabular}

motor support holds the motor from the back and it is clamped to the base-plate of the foundation at the bottom. The modal analysis of the motor support was carried out in isolation to find out the stiffnesses in horizontal and vertical direction. The data is acquired by exciting the motor support by impact hammer and measuring the response using accelerometers. The stiffnesses of the motor support are identified in horizontal and vertical direction using experimental data. The identified horizontal and vertical stiffnesses are $1.087 \times 10^{7}$ and $1.4 \times 10^{7} \mathrm{~N} / \mathrm{m}$. The mass of the back plate, of the motor support is used for identification of stiffnesses. The mass of the back plate is approximately $2.5 \mathrm{~kg}$.

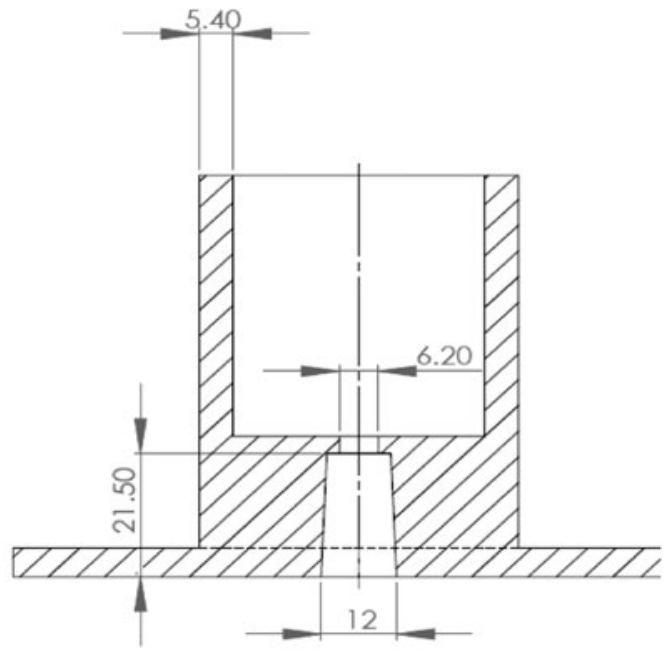

\subsection{Axial bearing support}

The axial bearing support is shown in the Fig. 9. The axial bearing support consists of two sets of leaf springs, one in the inner side and the other at the outer side. The axial bearing support was designed in such a way that it provides movement only in axial direction. The inner leaf springs provide guidance in only axial direction for the support structure whereas motion is achieved by outer leaf springs. The axial bearing is placed in between the bearing housing and the rotor, which in turn is attached to a high speed motor drive. The modal test of the bearing supports is carried out in isolation. The fundamental natural frequency of the bearing support is $3.1 \mathrm{~Hz}$ and stiffness of the bearing support is calculated and it comes out be $177 \mathrm{~N} / \mathrm{m}$.

\section{Axial bearing experimental setup}

In this section, the experimental setup for axial bearing identification is presented. All the components, which are discussed in previous sections, are assembled to form the complete experimental setup. The axial bearing experimental setup is shown in the Fig. 10. It can be noticed from Fig. 10 that the high speed motor is clamped to the support from behind, which in turn is bolted to the base plate of the foundation. The axial setup consists of a bearing 


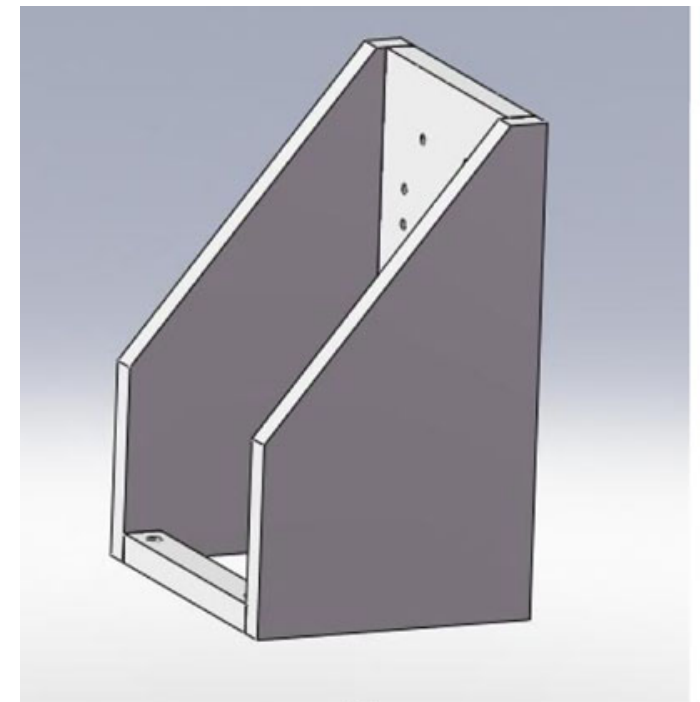

(a)

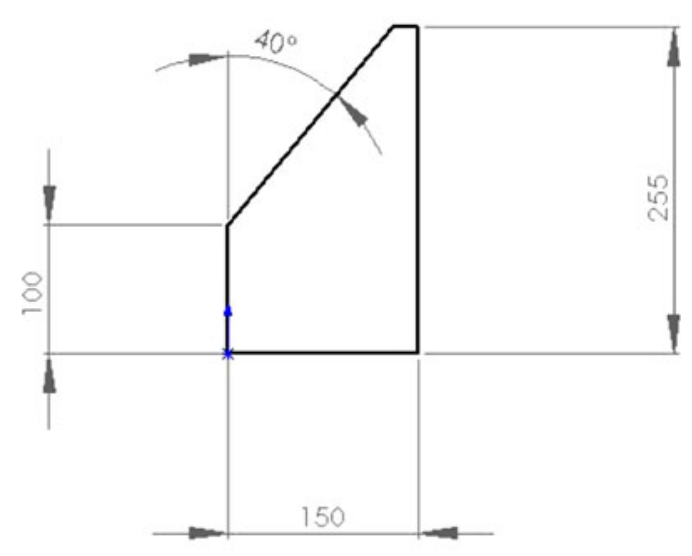

(b)

Fig. 8 a 3-D model and b side view of the motor-support

Fig. 9 a Isometric view and $\mathbf{b}$ pictorial view of axial-bearing support

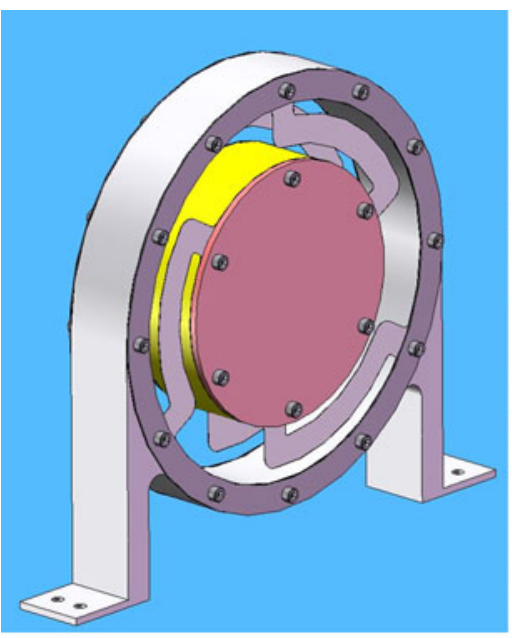

(a)

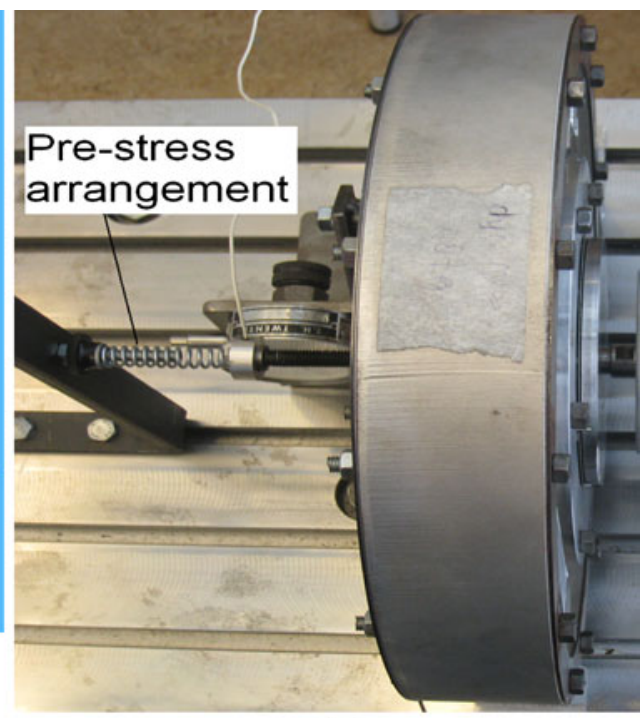

(b) support structure, in which the axial bearing housing is suspended. This suspension allows for movement in the axial direction only. The axial bearing is placed in between the bearing housing and the rotor, which in turn is attached to a high speed motor drive. The rotor is clamped to the motor on one end and on the other side the rotor disk is in contact with the axial bearing. The pre-stress on the axial bearing is applied by first screwing in the pre-stress adjuster until the force transducer reads a specified amount as shown in Fig. 10. After this, the dial gauge is set to zero, and the pre-stress is backed off until the displacement reads between upper and lower bound of tolerance band. This displacement is kept constant throughout the measurement session. The axial bearing is pressed between the rotor disc and the axial bearing support as shown in Fig. 11. To determine the eigenfrequencies and corresponding amplitudes of the axial 
Fig. 10 Axial bearing setup

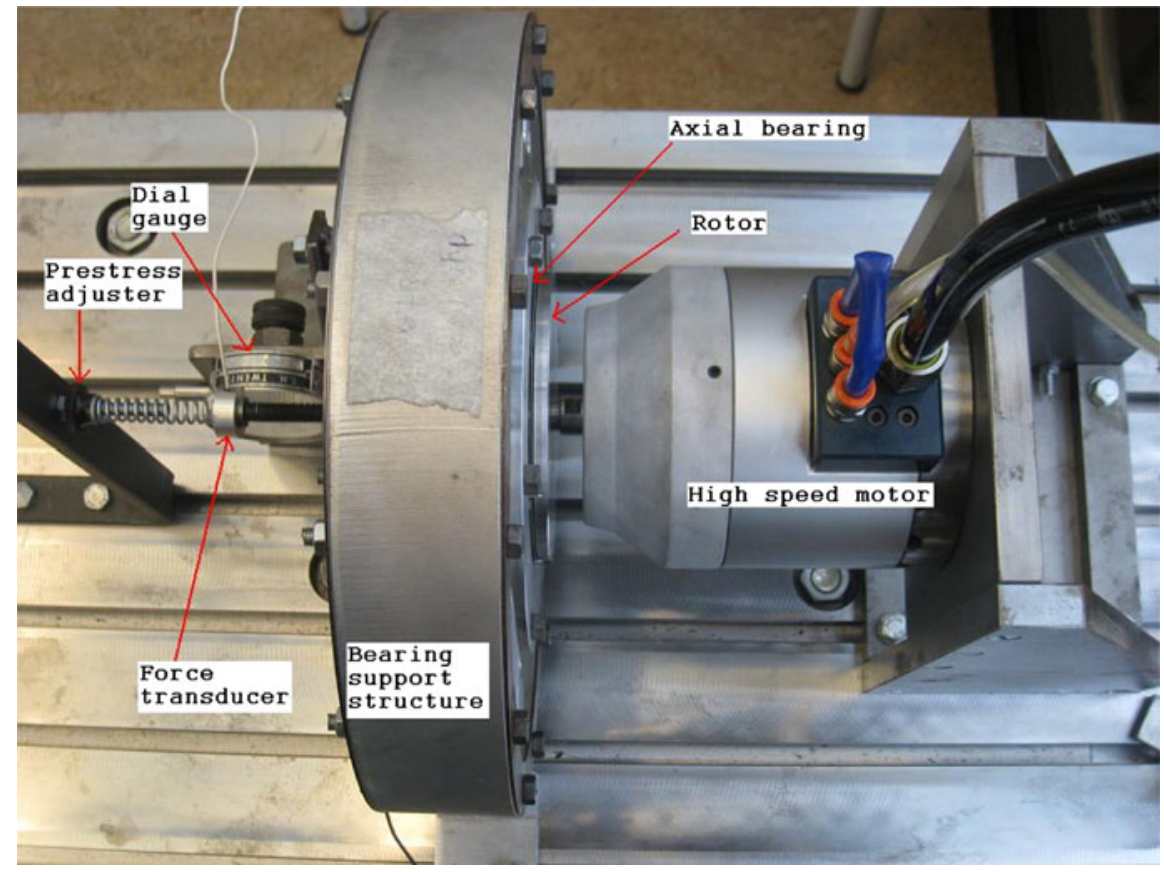

determining the axial bearing stiffness and damping characteristics. Several measurements have been carried out on the bearing structure, for RPM from 0 to 60,000 with 5,000 RPM increments, and for initial displacements of specified amounts. This means a set of 26 different conditions. For the 0 RPM case the rotor will be excited in axial direction by the aforementioned impact hammer, in all other cases the free response will be measured. Several fully static measurements will also be carried out using only the force transducer and dial gauge, to directly determine the force-displacement diagram of the bearing. For the non-static measurements, the FFT software measures the acceleration of the system 10 times in succession, and computes the average. The auto-spectrum of the signal is used to find the natural frequencies and their amplitudes in $\mathrm{dB}$. Repeatability of the results is investigated by performing all measurements twice on separate occasions.

The sub-structuring technique has been employed for identification of the bearing characteristics. Addition of components adds natural frequencies to the system. This can be illustrated by taking the example of a sub-structure of the experimental setup and complete axial bearing test rig. Experimentation is carried out on a sub-structure consisting of motor support, motor and rotor as shown in Fig. 12 and 


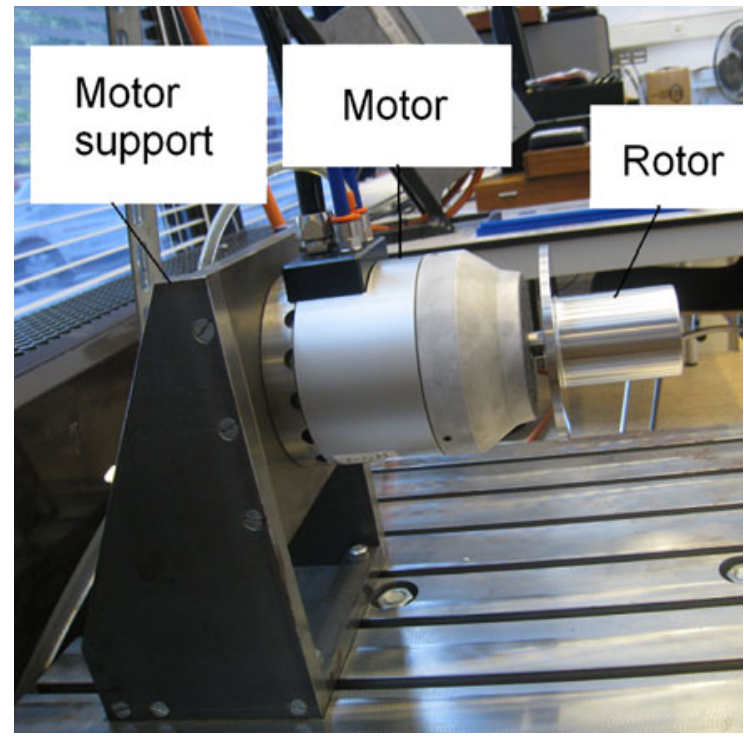

Fig. 12 Sub-structure consisting of motor support, motor and rotor

Table 2 Comparison of natural frequencies of sub-structure and complete structure

\begin{tabular}{llc}
\hline Mode number & $\begin{array}{l}\text { Motor }+ \text { support } \\
+ \text { rotor }(\mathrm{Hz})\end{array}$ & $\begin{array}{l}\text { Complete } \\
\text { system }(\mathrm{Hz})\end{array}$ \\
\hline 1. & 283 & 3.1 \\
2. & 332 & 85.5 \\
3. & & 282.8 \\
4. & & 337.1 \\
\hline
\end{tabular}

complete experimental setup. Because of the addition of the support and the axial bearing, there is addition of the natural frequencies in the system as given in the Table 2. It can be noticed from Table 2 that because of the introduction of the axial support and axial AFB, there is an introduction of two new natural frequencies at 3.1 and $85.5 \mathrm{~Hz}$. The first frequency $(3.1 \mathrm{~Hz})$ is equal to the natural frequency of the axial bearing support (which has already being identified during experimentation of the axial bearing support in isolation. Details are given in Sect. 3.4) and second frequency is due to the stiffness of the axial bearing.

The finite element of the rotor system is developed using 2D-beam elements. 2D-beam element has 6 degrees of freedom; each node has 3 degrees of freedom as shown in the Fig. 13. The stiffness properties of each component identified in the previous section are then included in the finite element model of the rotor to develop the complete model of the system. Initially, an arbitrary value of the bearing stiffness is assigned to the rotor-supportbearing system. The modal analysis of the finite element model is carried out to find its dynamic characteristics. The predicted dynamic characteristics of the finite element model are compared with the experimental data obtained, as given in the test plan section. The typical response at $15,000 \mathrm{rpm}$ speed is shown in the Fig. 14.

\section{Results}

As already discussed in the sub-structuring section, because of the stiffness of the axial bearing, there is an addition of the natural frequency in the system. This natural frequency is used to identify the stiffness of the axial bearing. For the case of the axial bearing, the response is measured up to $60,000 \mathrm{rpm}$ with the increments of 5,000 rpm. The data is then analyzed to obtain natural frequencies and damping ratios while maintaining a gap of specified amount as discussed in the test plan. The experimental natural frequencies and damping ratios are then used to identify stiffness and damping of the axial bearing. As discussed in the Sect. 3.4 the mode at a frequency of around $80 \mathrm{~Hz}$ is because of the axial bearing stiffness. For all readings, the mode near $80 \mathrm{~Hz}$ is considered for identification. The stiffness and damping of the axial bearing is identified using the inverse eigensensitivity method. The finite element model of the rotor bearing system and identification method is developed in MATLAB. The identified stiffness and damping values are given in Figs. 15 and 16 respectively. For plotting stiffness and damping plot shape-preserving curve has been used as it preserves monotonicity and the shape of the data. It can be observed from Fig. 15 that the stiffness value is very high at the static position of the rotor and the value of stiffness decreases with increase in speed of the rotor. It can be explained as during static condition, there is a contact between the bearing and the rotor because of that the stiffness is very high as the rotor picks up the speed a gas-film develops between the rotor and the bearing which causes the decrease in the stiffness. It can also be observed from Fig. 15 that the value of stiffness from 5,000 rpm till 45,000 rpm remains constant and after that there is a decrease in stiffness 
Fig. 13 2D-beam element

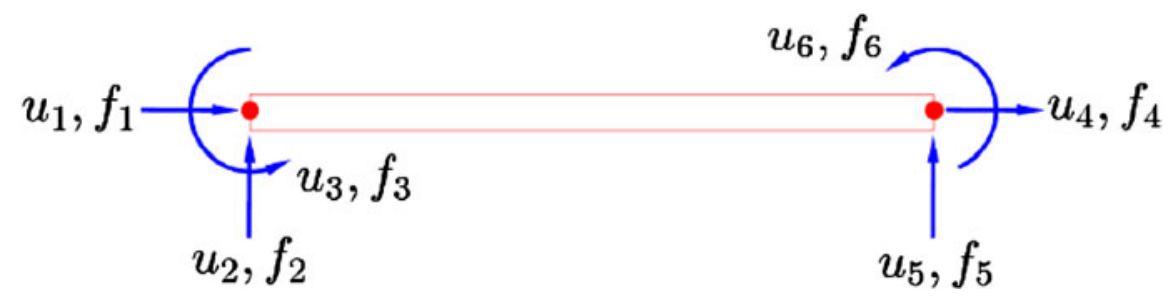

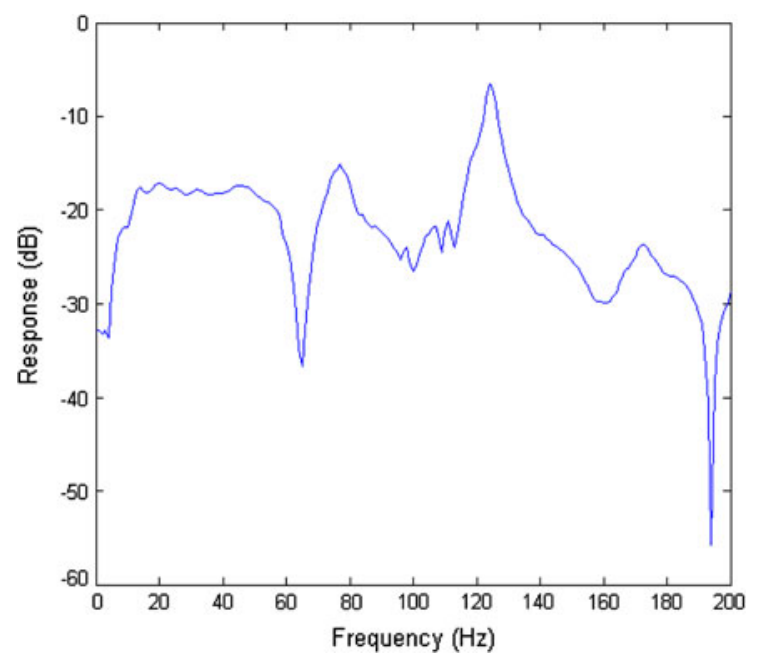

Fig. 14 Response at 15,000 rpm

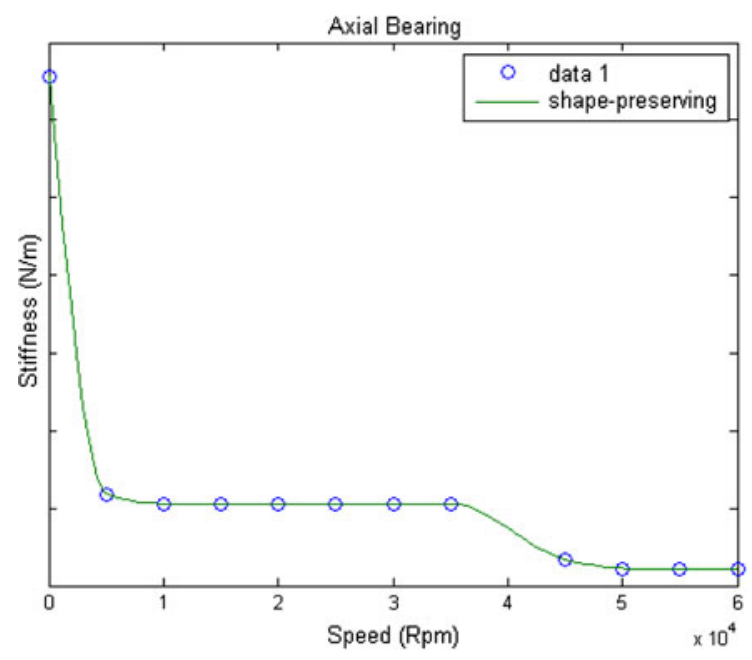

Fig. 15 Identified stiffness at various speed for axial bearing

at $45,000 \mathrm{rpm}$ and after that it again remains constant. This phenomenon can be due to transition from laminar to turbulent air flow because of very high speed. It can be observed from the identified damping plot as shown in Fig. 16 that at the static

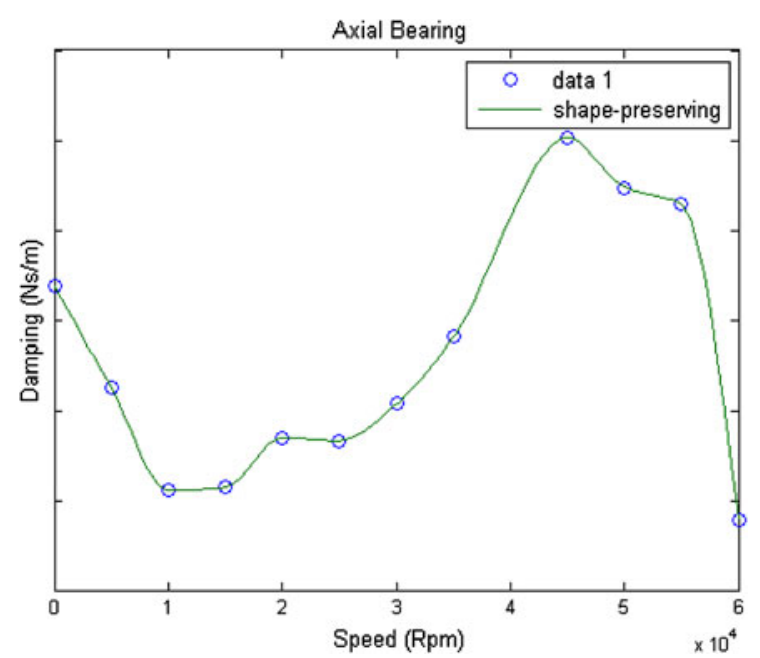

Fig. 16 Identified damping at various speed for axial bearing

condition damping value is very large because of the contact. As the speed increases the damping of the axial bearing decreases but again at high speed because of development of the gas film, there is an increase in the damping value.

\section{Conclusions}

In this paper, a new experimental setup is proposed. The proposed experimental setup uses a single axial AFB instead of conventional two AFB in rotorbearing system. Sub-structuring technique is used in the present work. In the substructuring technique, the structural characteristics of each component of the rotor-bearing-support system are identified in isolation and then identified structural characteristics of each component are used in developing the finite element model of the rotor-bearing-support system. The developed finite element model of the rotorbearing-support system is subsequently used for identification of structural characteristics of the axial AFB. A new identification algorithm for AFB is also 
developed. The developed method is based on the inverse eigensensitivity method and it uses eigendata for identification of the structural characteristics of the bearings. The developed algorithm is working well. From the identification results, it can be concluded that the stiffness value is very high at the static position of the rotor and the value of stiffness decreases with increase in speed of the rotor. It can be explained as during static condition, there is a contact between the bearing and the rotor because of that the stiffness is very high as the rotor picks up the speed a gas-film develops between the rotor and the bearing which causes the decrease in the stiffness. Similarly for damping, at static condition damping value is very large because of the contact. As the speed increases the damping of the axial bearing decreases but again at high speed because of development of the gas film, there is an increase in the damping value.

Acknowledgments The work presented in this paper was supported by research grant from Agentschap NL.

Open Access This article is distributed under the terms of the Creative Commons Attribution Noncommercial License which permits any noncommercial use, distribution, and reproduction in any medium, provided the original author(s) and source are credited.

\section{References}

Arora, V., Singh, S.P., Kundra, T.K.: Damped model updating using complex updating parameters. J. Sound Vib. 320, 438-451 (2009a)

Arora, V., Singh, S.P., Kundra, T.K.: Finite element model updating with damping identification. J. Sound Vib. 324, 1111-1123 (2009b)

Belforte, G., Raparelli, T., Viktorov, V., Trivella, A., Colombo, F.: An experimental study of high-speed rotor supported by air bearings: test rig and first experimental results. Tribol. Int. 39, 839-845 (2006)

Chen, J.H., Lee, A.C.: Estimation of linearized dynamic characteristics of bearing using synchronous response. Int. J. Mech. Sci. 37, 197-219 (1995)

DellaCorte, C., Valco, M.: Load capacity estimation of foil air Journal bearing for oil-free turbomachinery applications. STLE Tribol. Trans. 43, 795-801 (2000)

Edwards, S., Lee, A.W., Friswell, M.I.: Experimental identification of excitation and support parameters of flexible rotor-bearings-foundation system from a single run-down. J. Sound Vib. 232, 963-992 (2002)

Fox, R.L., Kapoor, M.P.: Rates of changes of eigenvalues and eigenvectors. AIAA J. 6, 2426-2429 (1968)

Heshmat, H., Ku, C.P.: Structural damping of self-acting compliant foil journal bearings. ASME J. Tribol. 116, 76-82 (1994)

Isomura, K., Tanaka, S., Togo, S., Esashi, M.: Development of high-speed micro-gas bearings for three dimensional micro-turbo machines. J. Micromech. Microeng. 15, 222227 (2005)

Jiang, P.L., Yu, L.: Identification of the oil-film dynamic coefficients in the rotor-bearing system with a hydrodynamic thrust bearing. J. Sound Vib. 236, 733-740 (2000)

Kim, D., Park, S.: Hydrostatic air-foil bearings: analytical and experimental investigation. Tribol. Int. 42, 413-425 (2009)

Kim, Y.H., Yang, B.S., Tan, A.C.C.: Bearing parameter identification of rotor-bearing system using cluster-based hybrid evolutionary algorithm. Struct. Multi. Option 33, 493-506 (2007)

$\mathrm{Ku}$, C.P.: An experimental and theoretical study of the dynamic structural stiffness in compliant foil journal bearings. In: ASME 14th Biennial Conference on Mechanical Vibration and Noise, Albuquerque, vol. 63, pp. 83-88 (1993)

$\mathrm{Ku}$, C.P., Heshmat, H.: Complaint foil bearing structural stiffness analysis, part II: experimental investigation. ASME J. Tribol. 115, 364-369 (1993)

$\mathrm{Ku}$, C.P., Heshmat, H.: Structural stiffness and coulomb damping in compliant foil journal bearing: theoretical considerations. STLE Tribol. Trans. 37, 525-533 (1994a)

$\mathrm{Ku}$, C.P., Heshmat, H.: Structural stiffness and coulomb damping in compliant foil journal bearing: Parametric studies. STLE Tribol. Trans. 37, 455-462 (1994b)

Lee, C.W., Hong, S.W.: Identification of bearing dynamic coefficients by unbalance response measurements. In: Proceedings of the Institution of Mechanical Engineering Conference, pp. 93-101 (1998)

Modak, S.V., Kundra, T.K., Nakra, B.C.: Comparative study of model updating methods using simulated experimental data. Comput. Struct. 80, 437-447 (2000)

Rubio, D., San Andrés, L.: Bump-type foil bearing structural stiffness: experimentation and predictions. J. Eng. Gas Turbine Power 128, 653-660 (2006)

Salehi, M., Heshmat, H., Walton, J.: On the frictional damping characterization of compliant bump foils. ASME Trans. 125, 804-813 (2003)

San Andrés, L., Kim, T.H.: Forced nonlinear response of gas foil bearing supported rotors. Tribol. Int. 704, 413-715 (2008)

Tiwari, R., Lee, A.W., Friswell, M.I.: Identification of speed-dependent bearing parameters. J. Sound Vib. 254, 967-986 (2002) 